\title{
A prospective self-controlled study on shortening the time before taking delayed radiographs with iodized oil hysterosalpingography
}

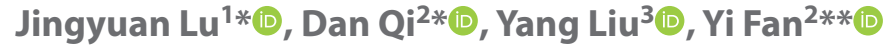 \\ ${ }^{1}$ Department of Radiological Intervention, The Women's Hospital of Nanjing Medical University, Nanjing, Jiangsu, China \\ ${ }^{2}$ Department of Traditional Chinese Medicine, The Women's Hospital of Nanjing Medical University, Nanjing, Jiangsu, China \\ ${ }^{3}$ Department of Obstetrics and Gynecology, The Women's Hospital of Nanjing Medical University, Nanjing, Jiangsu, China
}

*Jingyuan Lu and Dan Qi contributed equally to this article

**Yi Fan is co-corresponding author

\begin{abstract}
Objectives: To verify the feasibility of walking to shorten the time before obtaining delayed radiographs after iodized oil hysterosalpingography (HSG).

Material and methods: One hundred women with infertility were selected for HSG from June 2018 to December 2018 at the Women's Hospital of Nanjing Medical University; the subjects were randomly divided into walking and control groups. The walking group was required to walk more than 12,000 steps within 6 hours after HSG, while the control group was prohibited from performing high-intensity exercise. The degree of pelvic adhesion was diagnosed with delayed radiographs acquired at 6 and 24 hours, and the diagnostic consistency of the radiographs at the two time points was evaluated.

Results: No significant difference was observed in the baseline data between groups $(p>0.05)$. The delayed radiograph results in the walking group showed good agreement $(p=0.255>0.05$, Kappa value $0.781>0.75)$, while those in the control group showed general agreement $(p=0.002<0.05$, Kappa value $0.493>0.40<0.75)$.

Conclusions: The time for acquiring delayed radiographs can be shortened by instructing patients to walk after HSG. This method improves the diagnostic efficiency of lodized oil, saves time and costs, and may contribute to the popularization of HSG for female infertility screening, while offering good clinical application prospects.
\end{abstract}

Key words: hysterosalpingography; lodized oil; delayed radiographs; pelvic adhesions; self-controlled study

Ginekologia Polska 2020; 91, 11: 655-660

\section{INTRODUCTION}

Hysterosalpingography (HSG), a long-standing diagnostic imaging technology, plays an important role in preliminary screening for female infertility due to its simplicity, convenience, minimal trauma and image quality. Oily contrast agents have obvious advantages in showing details of uterine tubal lesions and pelvic adhesions. With continuous improvements in equipment and technology, the risk of serious complications such as pulmonary embolism is decreasing. Recent studies have shown that lipiodol contrast agents improve patient pregnancy rates [1], which has increased interest in HSG. However, delayed films should be taken at 24 hours after initial HSG to evaluate the presence of pelvic adhesions, which significantly reduces diagnostic efficiency and speed and increases the time cost for pa- tients. Especially for nonlocal patients, it also increases the cost of transportation or accommodation, which greatly hinders the popularization of iodized oil HSG. The aim of this study was to promote pelvic iodized oil diffusion through appropriate exercise after HSG. This method could shorten the time before delayed films can be obtained, improve diagnostic efficiency, and reduce the time and economic costs for patients without affecting diagnostic accuracy.

\section{MATERIAL AND METHODS \\ Clinical material}

From June 2018 to December 2018, one hundred female infertility patients who underwent HSG at the Women's Hospital of Nanjing Medical University, aged 33.54 \pm 6.41 years, with an infertility duration of $4.80 \pm 3.08$ years, were se- 
lected. All subjects were randomly divided into walking and control groups ( $n=50$ each). The inclusion, exclusion and removal criteria were shown in Table 1. And this study was approved by the Medical Ethics Committee of The Women's Hospital of Nanjing Medical University.

\section{Equipment and drugs}

A Shimadzu Digital Gastrointestinal System (FLEXAVISION, Japan) was used for imaging during the HSG procedure. Shida disposable hysterography tubes (type 12B, China) were the main consumable items used. Papaver Ethyl lodine Oil Injection (Hengrui Medicine, China) was used as a contrast agent.

\section{HSG operation process}

The procedure was performed 3 to 7 days after menstruation. First, the perineum was disinfected, and surgical towels were spread out. Second, an angiographic catheter was inserted into the uterine cavity under $X$-ray guidance. Third, the balloon was filled to seal the cervical opening. Fourth, iodized oil was gently injected into the uterine cavity. Images of uterine filling, tubal filling and pelvic overflow were recorded. Finally, the balloon was withdrawn, and the catheter was removed.

\section{Exercise guidance and delayed radiographs obtained}

After HSG, the researchers instructed the walking group patients to walk at a prescribed intensity to accelerate pelvic contrast medium diffusion. These subjects were instructed to walk more than 12,000 steps within 6 hours after HSG. The control subjects were asked to maintain normal daily activity intensity and avoid strenuous exercise post-HSG. "WeChat" (Tencent Inc, China) was used to count the steps walked and quantify exercise intensity post-HSG.

Delayed radiographs were obtained for the two groups at $6 \pm 0.5$ and $24 \pm 2$ hours post-HSG to evaluate pelvic dispersion of the contrast material.

\section{Randomization}

The delayed radiographs taken at 6 and 24 hours post-HSG were randomly assigned to five diagnostic physicians ( $A$ to $E$ ) for evaluation of the degree of pelvic adhesion, which was divided into four grades (grade 0 to 3 ), as shown in Table 2.

All operators, imaging technicians and imaging diagnosticians involved in the clinical trial had more than 5 years of experience in HSG-related work. Before initiation of this

\begin{tabular}{|c|c|}
\hline & Criteria \\
\hline Inclusion & $\begin{array}{l}\text { 1. Women aged } 21 \text { to } 45 \text { years } \\
\text { 2. Conformity with HSG indications } \\
\text { 3. Voluntary participation in the trial with informed consent }\end{array}$ \\
\hline Exclusion & $\begin{array}{l}\text { 1. History of iodine allergy, hyperthyroidism, or thyroid tumors } \\
\text { 2. Trichomonas vaginitis, candida vaginitis, vaginal secretion cleanliness over II degrees } \\
\text { 3. Acute/subacute pelvic inflammation, chronic pelvic inflammation, active pelvic/uterine/fallopian tuberculosis } \\
\text { 4. Uterine or cervical bleeding } \\
\text { 5. Less than } 6 \text { months postpartum or uterine cavity operation less than } 4 \text { weeks } \\
\text { 6. Infertility due to lack of ovulation } \\
\text { 7. Copulation or vaginal administration of semen within } 3 \text { days before HSG } \\
\text { 8. Body temperature of higher than } 37.5^{\circ} \mathrm{C} \text { on the day of HSG } \\
\text { 9. Inability to diagnose the degree of pelvic adhesion because iodized oil could not flow into the pelvic cavity through any side of the } \\
\text { fallopian tube after intrauterine injection }\end{array}$ \\
\hline Removal & $\begin{array}{l}\text { 1. Incorrect diagnosis at admission } \\
\text { 2. HSG was immediately terminated because of serious complications, such as venous/lymphatic reflux or allergic reaction } \\
\text { 3. The subject asked to withdraw from the study } \\
\text { 4. Researchers deemed a subject no longer suitable for inclusion in the study for medical safety reasons } \\
\text { 5. The patient failed to fulfill or seriously violated the research plan }\end{array}$ \\
\hline
\end{tabular}

Table 2. Imaging grading of pelvic adhesions

\begin{tabular}{l|l}
\hline Classification & Diagnostic criteria \\
\hline Grade 0 (No clear pelvic adhesions) & Wide and uniform contrast agent distribution in the pelvic cavity \\
\hline Grade 1 (Mild pelvic adhesions) & $\begin{array}{l}\text { The pelvic contrast medium diffusion range was slightly smaller than normal, and the distribution was } \\
\text { generally uniform }\end{array}$ \\
\hline Grade 2 (Moderate pelvic adhesions) & The contrast medium diffusion range was obviously limited, and the medium was strongly localized \\
\hline Grade 3 (Severe pelvic adhesions) & $\begin{array}{l}\text { The contrast medium was concentrated in the pelvic region and had not disseminated, resulting in a mass } \\
\text { image }\end{array}$ \\
\hline
\end{tabular}


study, a coordination meeting was held to standardize the processes followed by the operation and technical groups and to standardize the pelvic adhesion evaluation criteria used by the diagnostic group.

The MedSci medical randomization tool was used for randomization. One hundred subjects (50 each in the walking and control groups) were included, with 200 delayed film images.

\section{Blinding}

All diagnosing doctors were blinded. After the quality controllers removed the subjects' identifying information and times that the imaging data were obtained, the delayed films taken 6 and 24 hours post-HSG in the walking and control groups were randomly sent to the five diagnostic physicians. These physicians evaluated the degree of pelvic adhesiveness (grade 0 to 3 ) on the pelvic images. To eliminate interference of the diagnostician's residual image memory on the diagnosis (for example, if the same subject was assigned to the same diagnostician at different times after randomization), the interval between reading the two sets of imaging data was required to be longer than 14 days.

\section{Data analysis}

IBM SPSS version 20 software was used to analyze the data collected in this trial. Measurement data are expressed as $\bar{x} \pm s$, and numerical data are expressed as percentages (\%). One-way ANOVA was used to compare the normally distributed measurement data that passed the homogeneity of variance test. The rank sum test was used to compare the non-normally distributed grade data and measurement data that did not pass the homogeneity of variance test. The chi-square test was used for non-grade count data. A value of $\mathrm{P}<0.05$ indicated a significant difference.

The 6- and 24-hour standard delayed radiographs of the walking and control groups were compared. McNemar-Bowker test was used to evaluate the statistical difference between 6-hour and 24-hour delayed radiograph. And the diagnostic consistency between the two groups was evaluated using the Kappa test. If the Kappa value was higher than 0.75 , the diagnosis based on the 6-hour delayed film was considered consistent with that of the traditional 24-hour delayed film; therefore, the 6-hour film could be used for diagnosis instead of the 24-hour film. If the Kappa value was lower than 0.40 , the diagnostic consistency between the 6- and 24-hour films was considered poor. If the Kappa value was between 0.40 and 0.75 , the diagnostic consistency between the 6- and 24-hour films was intermediate, making it necessary to analyze the clinical trial design or to increase the sample size for further observation.

\section{RESULTS}

No significant differences in age, number of pregnancies, production time or number of years of infertility were observed between the walking and control groups ( $p>0.05)$, as shown in Table 3.

There were no significant differences in the histories of pelvic inflammation, endometriosis, appendicitis, tubal pregnancy, cesarean section and other pelvic operations between the walking and control groups ( $p>0.05)$, as shown in Table 4.

The McNemar-Bowker test showed no significant difference between the 6-and 12-hour data in the walking group $(p=0.255>0.05)$. There was no significant difference between the results of the 6-and 24-hour delayed radiographs in the diagnosis of pelvic adhesion. The Kappa value was 0.781 , which exceeded 0.75 . The results suggested that the diagnosis of pelvic adhesion based on the 6-hour delayed radiograph was consistent with that based on the traditional 24-hour delayed radiograph. Therefore, it is feasible to use a 6-hour delayed film instead of a 24-hour delayed radiograph to diagnose pelvic adhesion.

According to the McNemar-Bowker test, $p=0.002<0.05$ for the 6- and 24-hour radiographs in the control group. A significant difference was observed between the 6- and 24-hour delayed radiographs in the diagnosis of pelvic adhesion. The Kappa coefficient was 0.493 , which was between 0.40 and 0.75 . For the control group, the diagnosis of pelvic adhesion based on the 6-hour delayed radiograph was somewhat consistent with that based on the 24-hour delayed radiograph. Therefore, the evidence did not support replacing the diagnosis based on a 24-hour delayed radiograph with that based on a 6-hour delayed radiograph.

\section{DISCUSSION}

According to recent reports, the incidence of female infertility in the general population ranges from 9 to $18 \%$

\begin{tabular}{|l|l|l|l|l|}
\hline Table 3. Comparison of measurement indexes between the two groups of subjects $(\overline{\mathbf{x}} \pm \mathbf{s})$ \\
\hline Measurement indexes & Walking group $(\mathbf{n}=\mathbf{5 0})$ & Control group $(\mathbf{n}=\mathbf{5 0})$ & t value & p value \\
\hline Age (years) & $34.52 \pm 6.57$ & $32.56 \pm 6.16$ & 1.539 & 0.127 \\
\hline Number of pregnancies & $1.72 \pm 1.11$ & $1.42 \pm 1.11$ & 1.354 & 0.179 \\
\hline Production times & $0.80 \pm 0.61$ & $0.78 \pm 0.68$ & 0.155 & 0.877 \\
\hline Number of years of infertility (years) & $4.82 \pm 2.90$ & $4.78 \pm 3.27$ & 0.065 & 0.949 \\
\hline
\end{tabular}


Table 4. Comparison of count data between the two groups of subjects

\begin{tabular}{|c|c|c|c|c|c|c|}
\hline \multirow{2}{*}{ Count indexes } & \multicolumn{2}{|c|}{ Walking group $(n=50)$} & \multicolumn{2}{|c|}{ Control group $(n=50)$} & \multirow{2}{*}{$x^{2}$} & \multirow{2}{*}{ p value } \\
\hline & Cases & Percentage (\%) & Cases & Percentage (\%) & & \\
\hline Pelvic infection & & & & & 0.219 & 0.640 \\
\hline Yes & 37 & 74.0 & 39 & 78.0 & & \\
\hline No & 13 & 26.0 & 11 & 22.0 & & \\
\hline Endometriosis & & & & & 0.298 & 0.585 \\
\hline Yes & 43 & 86.0 & 41 & 82.0 & & \\
\hline No & 7 & 14.0 & 9 & 18.0 & & \\
\hline Appendicitis & & & & & 0.000 & 1.000 \\
\hline Yes & 46 & 92.0 & 47 & 94.0 & & \\
\hline No & 4 & 8.0 & 3 & 6.0 & & \\
\hline Tubal pregnancy & & & & & 0.271 & 0.603 \\
\hline Yes & 42 & 84.0 & 40 & 80.0 & & \\
\hline No & 8 & 16.0 & 10 & 20.0 & & \\
\hline Cesarean section & & & & & 0.480 & 0.488 \\
\hline Yes & 39 & 78.0 & 36 & 72.0 & & \\
\hline No & 11 & 22.0 & 14 & 28.0 & & \\
\hline Other pelvic surgery & & & & & 0.233 & 0.629 \\
\hline Yes & 40 & 40.0 & 38 & 76.0 & & \\
\hline No & 10 & 20.0 & 12 & 24.0 & & \\
\hline
\end{tabular}

[2], and the incidence is increasing [3]. With the liberalization of the national fertility policy, the diagnosis and treatment of female infertility has become a prominent clinical issue in China [4]. The main causes of female infertility are related to the fallopian tubes [5], ovaries [6] and uterus [7]. In addition, pelvic adhesion [8] is considered an important cause of female infertility. Among the above factors, the diagnosis of ovarian factor infertility mainly relies on laboratory examinations to detect and analyze reproductive endocrine hormones [9]. Tubal factor, uterine factor and pelvic adhesion infertility can be diagnosed by hysteroscopy, laparoscopy [10] and imaging [11] because they can cause morphological changes.

The main imaging methods currently used to diagnose female infertility are hysterosalpingo contrast sonography (HyCoSy) [12], MR [13] and HSG [14]. Among these methods, HSG has the advantages of minimal trauma, low cost, convenience, clear results, and the ability to show tubal, uterine and pelvic adhesion at the same time; thus, it is widely used for assessing female infertility.

According to the different contrast agents used, HSG can be further divided into iodine water radiography and iodine oil radiography. The advantages of iodine hydrography are mainly safety (no risk of serious complications such as pulmonary embolism) and convenience (short time prior to the delayed radiograph). However, iodized oil radiography has obvious advantages in the level of detail shown of uter- ine and fallopian tube lesions and pelvic adhesions. In addition, recent studies have revealed that lipiodol angiography can improve pregnancy rates due to its immunomodulatory effects [15]. Therefore, HSG with iodized oil has attracted the attention of clinicians and has been increasingly applied for the screening of female infertility.

Inflammation, surgery and pelvic endometriosis are common causes of pelvic adhesions, which are closely related to female infertility [16]. Different degrees of pelvic adhesions have varying impacts on the patency of fallopian tubes and the prognosis of treatment [17]; thus, increased attention should be paid to the diagnosis of pelvic adhesions.

The fimbriated extremity of fallopian opens in the peritoneal cavity. During HSG, the contrast agent is injected into the uterine cavity through the cervix via a contrast catheter, moves into the fallopian tube cavity, and finally exits into the peritoneal cavity through the opening of the fimbriated extremity of fallopian. After the contrast agent enters the peritoneal cavity, it continuously diffuses to distant locations due to body movement and intestinal peristalsis. If pelvic peritoneal adhesions exist, the diffusion process is hindered, and the contrast agent accumulates in the region with adhesions. Imaging diagnosticians evaluate pelvic adhesions by observing contrast media diffusion. When a water-based contrast agent is used for HSG, the delayed radiograph can be obtained after approximately $15 \mathrm{~min}$ utes [18]. As the dispersion and absorption of water-based 
contrast agents occur very quickly, the details of the diffusion image are difficult to capture, making the diagnostic value of the test for pelvic adhesions relatively low. The diagnostic accuracy of HSG for pelvic adhesions is much higher when oil-based contrast agents are used than when water-based contrast agents are used because of the slow diffusion and absorption of the former. Therefore, to ensure the complete diffusion of iodine oil throughout the pelvic cavity, a longer interval of approximately 24 hours is needed before re-examination [19].

After the oil contrast agent enters the peritoneal cavity from the opening of the fimbriated extremity of fallopian, it spreads continuously around the spillover point and eventually reaches diffusion equilibrium, i.e., the point at which the diffusion range no longer expands. This process is usually completed approximately 24 hours after iodized oil injection. The diffusion rate and range of iodized oil are not only related according to the degree of pelvic adhesion but also affected by body movement and intestinal peristalsis. This principle is similar to that affecting cancer patients, who are required to change their posture frequently after intraperitoneal infusion chemotherapy to promote the uniform distribution of the drugs in their abdominal cavities [20]. This phenomenon provided us with an idea: after iodized oil angiography, increasing patient movement could accelerate the spread of iodized oil, thereby reducing the time needed to achieve diffusion equilibrium and ultimately shortening the interval before the delayed radiograph can be obtained.

Although MRI, contrast-enhanced ultrasound and laparoscopy are all used to diagnose uterine and fallopian tubal infertility, HSG still plays an irreplaceable role, especially in screening. Compared to water-based contrast media, oil-based contrast media have obvious advantages when used for diagnosis and could promote pregnancy by modulating immunity after HSG. Therefore, the use of HSG with oil-based contrast medium has become increasingly widespread in recent years. With the improvement of surgical instruments, operative technology and the technology involved in contrast agent production, the risk of serious complications of lipiodol radiography has significantly decreased, and there is now no obvious safety disadvantage compared to iodized water radiography. Currently, as the pace of life is clearly accelerating, the 24-hour interval before obtaining a delayed radiograph has become an important hindrance to the further promotion of HSG with oil-based contrast medium.

The shortcomings of our study included the small sample size and use of only one additional time point for the delayed radiograph (6 hours), which prevented precise determination of the time required to reach dispersion equilibrium. Additionally, the stride length, stride frequency and walking duration differed among the subjects. Simply analyzing the number of steps did not accurately reflect the exercise intensity.

The results of this exploratory research, which aimed to maintain diagnostic accuracy, revealed that the diagnostic efficiency of HSG with an oil-based contrast agent could be improved and the time required could be reduced by instructing patients to exercise appropriately. Especially for nonlocal patients, this new method could reduce the amount of time needed for delayed radiographs to less than one day, reducing the costs associated with transportation and accommodation. These findings suggest that HSG is clinically applicable and should be further promoted for use in female infertility screening.

\section{Conflicts of interest}

No conflict of interests to declare.

\section{REFERENCES}

1. Dreyer K, van Rijswijk J, Mijatovic V, et al. Oil-Based or Water-Based Contrast for Hysterosalpingography in Infertile Women. N Engl J Med. 2017; 376(21): 2043-2052, doi: 10.1056/NEJMoa1612337, indexed in Pubmed: 28520519.

2. Aghajanova L, Hoffman J, Mok-Lin E, et al. Obstetrics and Gynecology Residency and Fertility Needs. Reprod Sci. 2017; 24(3): 428-434, doi: 10.1177/1933719116657193, indexed in Pubmed: 27368879.

3. Zhou Z, Zheng D, Wu H, et al. Epidemiology of infertility in China: a population-based study. BJOG. 2018; 125(4): 432-441, doi: 10.1111/14710528.14966, indexed in Pubmed: 29030908.

4. Aketayeva A, Khamidullina Z, Akhmetova Z, et al. Diagnosis and Treatment of Female Infertility Is One of the Major Problems in Modern Gynecology. Iran J Public Health. 2018; 47(1): 135-137, indexed in Pubmed: 29318130.

5. Chua SuJ, Akande VA, Mol BW. Surgery for tubal infertility. Cochrane Database Syst Rev. 2017; 1: CD006415, doi: 10.1002/14651858.CD006415. pub3, indexed in Pubmed: 28112384.

6. Szafarowska M, Jerzak M. [Ovarian aging and infertility]. Ginekol Pol. 2013; 84(4): 298-304, doi: 10.17772/gp/1580, indexed in Pubmed: 23700864.

7. Ait Benkaddour Y, Gervaise A, Fernandez H. [Which is the method of choice for evaluating uterine cavity in infertility workup?]. J Gynecol Obstet Biol Reprod (Paris). 2010; 39(8): 606-613, doi: 10.1016/j. jgyn.2010.08.004, indexed in Pubmed: 20870363.

8. Tang LS, Xu J, Tang HY. Correlations of abnormal ultrasound audio-visual images of ovarian cortex surface and pelvic adhesion in infertile patients. Clin Exp Obstet Gynecol. 2014;41(2):160-162, doi: 10. 12891/ceog15932014, indexed in Pubmed. ; 24779243, doi: 10.12891/ceog15932014.

9. Tobler KJ, Shoham G, Christianson MS, et al. Use of anti-mullerian hormone for testing ovarian reserve: a survey of 796 infertility clinics worldwide. J Assist Reprod Genet. 2015; 32(10): 1441-1448, doi: 10.1007/s10815-015-0562-7, indexed in Pubmed: 26347341.

10. Zhang E, Zhang Y, Fang Li, et al. Combined hysterolaparoscopy for the diagnosis of female infertility: a retrospective study of 132 patients in china. Mater Sociomed. 2014; 26(3): 156-157, doi: 10.5455/msm.2014.26.156157, indexed in Pubmed: 25126006.

11. Steinkeler JA, Woodfield CA, Lazarus E, et al. Female infertility: a systematic approach to radiologic imaging and diagnosis. Radiographics. 2009; 29(5): 1353-1370, doi: 10.1148/rg.295095047, indexed in Pubmed: 19755600.

12. Hong $Q$, Cai $R$, Chen $Q$, et al. Three-Dimensional HyCoSy With Perfluoropropane-Albumin Microspheres as Contrast Agents and Normal Saline Injections Into the Pelvic Cavity for Morphological Assessment of the Fallopian Tube in Infertile Women. J Ultrasound Med. 2017; 36(4): 741-748, doi: 10.7863/ultra.16.03041, indexed in Pubmed: 28150322.

13. Cipolla V, Guerrieri D, Pietrangeli D, et al. Role of 3.0 Tesla magnetic resonance hysterosalpingography in the diagnostic work-up 
of female infertility. Acta Radiol. 2016; 57(9): 1132-1139, doi: 10.1177/0284185115617351, indexed in Pubmed: 26663038.

14. Ledbetter KA, Shetty M, Myers DT. Hysterosalpingography: an imaging Atlas with cross-sectional correlation. Abdom Imaging. 2015; 40(6): 1721-1732, doi: 10.1007/s00261-014-0284-9, indexed in Pubmed: 25389063.

15. Yun AJ, Lee PY. Enhanced fertility after diagnostic hysterosalpingography using oil-based contrast agents may be attributable to immunomodulation. AJR Am J Roentgenol. 2004; 183(6): 1725-1727, doi: 10.2214/ajr.183.6.01831725, indexed in Pubmed: 15547218.

16. Imudia AN, Kumar S, Saed GM, et al. Pathogenesis of Intra-abdominal and pelvic adhesion development. Semin Reprod Med. 2008; 26(4): 289-297, doi: 10.1055/s-0028-1082387, indexed in Pubmed: 18756406.

17. Hou HY, Chen YQ, Chen X, et al. Related factors associated with pelvic adhesion and its influence on fallopian tube recanalization in infertile patients. Zhonghua Fu Chan Ke Za Zhi. 2012:47(11):823-828, doi: 10.1007/s11783-011-0280-z, indexed in Pubmed: 23302122.

18. Olawale BB, Ademola AO, Gbadebo AG. Tubal abnormalities in patients with intrauterine adhesion: evaluation using hysterosalpingography. Ann Afr Med. 2014; 13(4): 179-183, doi: 10.4103/1596-3519.142288, indexed in Pubmed: 25287031.

19. Tan Y, Zheng S, Lei W, et al. Ethiodized poppyseed oil versus ioversol for image quality and adverse events in hysterosalpingography: a prospective cohort study. BMC Med Imaging. 2019; 19(1): 50, doi: 10.1186/s12880-019-0346-0, indexed in Pubmed: 31234782.

20. Lu C, Li L, Luo Z, et al. Clinical efficacy of type-B ultrasound-guided intraperitoneal hyperthermic chemoperfusion combined with systemic chemotherapy in advanced gastric cancer patients with malignant ascites. Neoplasma. 2016;63(2):299-303, doi: 10.4149/217_150622N345, indexed in Pubmed: 26774152. 